\title{
Cancer Magnitude in Elderly Indian Women, an Experience from Regional Cancer Centre, India
}

\author{
Ravi Kiran Pothamsetty ${ }^{*}$, Radha Rani Ghosh ${ }^{2}$ and Baby Paul Thaliath ${ }^{3}$ \\ ${ }^{1}$ Department of Radiation Oncology, Regional Cancer Centre, KNM Hospital, India \\ ${ }^{2}$ Department of Radiation Oncology, Regional Cancer Centre, KNM Hospital, India \\ ${ }^{3}$ Department of Radiation Oncology, Regional Cancer Centre, KNM Hospital, India
}

*Corresponding author: Ravi Kiran Pothamsetty, Department of Radiation Oncology, Regional Cancer Centre, KNM Hospital, Allahabad, India

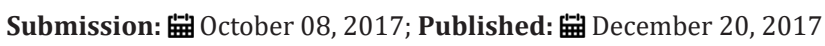

\begin{abstract}
According to WHO, older or elderly population referred to age $\geq 60$ years. The social life, economic stability and psycho-emotional domains were disturbed when an individual diagnosed with cancer. The present study attempted to analyze the prevalence of cancer in elderly Indian women reported to Regional Cancer Centre, India and review the literature to have an insight of cancer trends in Indian scenario. The retrospective analytical study was carried out in Department of Radiation Oncology, RCC, Allahabad, India. The study included 2215 cases of elderly women of age $\geq 60$ years from January 2013 to January 2016. Data were analyzed using SPSS statistical package version 14. Quantitative data were presented as median and standard deviation. Qualitative data were expressed as frequency and percentages. The total number of cancer cases in elderly women accounted for $31.25 \%$ as per the hospital based registry during the 3 year study period. The disease pattern has shown different trend compared to younger adults. Cancer cervix being the most common age group among females as per the hospital registry. Gall bladder cancers and breast cancers were the second most common cancers in elderly women and adults (20-59 years) respectively. Overall cancer cervix being the most common malignancy followed by gall bladder cancer in females in the region. Limited knowledge and misconceptions were prevailed among the participants. Collaboration with multiple cancer registries, hospitals and tertiary centers' nationwide can design a highly organized framework among elderly women with cancer which directs for potential future screening and detection programmes. Further research in gall bladder malignancy for early detection will reduce the mortality rates significantly. The article can be helpful for potential future research and directions.
\end{abstract}

Keywords: Cancer; Elderly; Women; India; Prevalence; Breast; Cervix; Trend; Framework; Registry

Abbreviations: HNC-Head and Neck Cancers; RCC-Regional Cancer Centre; AJCC-American Joint Committee on Cancer; FIGO- International Federation of Gynecology and Obstetrics (Fédération Internationale de Gynécologie et d’Obstétrique)

\section{Introduction}

India has experienced drastic transition in the population burden with rapid rise in the elderly population [1]. The projections depicted there will be a significant rise in the elderly population from $7.5 \%$ in 2010 to $11.1 \%$ in 2025 [2]. According to WHO, older or elderly population referred to age $\geq 60$ years [3]. Older women face a lot of age related issues which can impact the psychological and emotional balance in their lives. Cancer is one of the most predominant non communicable diseases in India, being responsible for high scale mortality and morbidity. The numbers of cancer cases were on rise making it as a serious public health concern. As per ICMR report, the estimated new cases and deaths projected up to 17.3 lakhs and over 8.8 lakhs respectively by 2020 [4]. The social life, economic stability and psycho-emotional domains were disturbed when an individual diagnosed with cancer. The journey to conquer cancer is a long way which requires courage and support to battle the deadly disease. The present study attempted to analyze the prevalence of cancer in elderly
Indian women reported to Regional Cancer Centre and review the literature to have an insight of cancer trends in Indian scenario.

\section{Objectives}

The study has set the following objectives with regards to-

a) Prevalence of cancer in elderly women.

b) Disease patterns among elderly women.

c) Age group at the time of presentation of the disease.

d) Knowledge and attitude of elderly women towards cancer.

\section{Materials and Methods}

The retrospective analytical study was carried outin Department of Radiation Oncology, RCC, Allahabad, India. The cancer statistics, trends, patient demographics, tumor characteristics, and other relevant information were recorded and documented in detail. The 
study included all elderly women of $\geq 60$ years during 3 years period from January 2013 to January 2016. The inclusion criteria included female sex $\geq 60$ years and willing to participate in the study. In our study a total of 2215 cases were enrolled. Imaging modalities such as Ultrasound, Chest X-Ray, CT scan, MRI wherever required were performed. Complete blood count with liver and kidney function tests and confirmation by biopsies were undertaken. Staging was done as per AJCC 2008. FIGO staging 2008 was applied for Gynecological malignancies. Tailored therapy as per the individual needs, stage, site and histology was rendered. Data were analyzed using SPSS statistical package version 14. Quantitative data were presented as median and standard deviation. Qualitative data were expressed as frequency and percentages.

\section{Results}

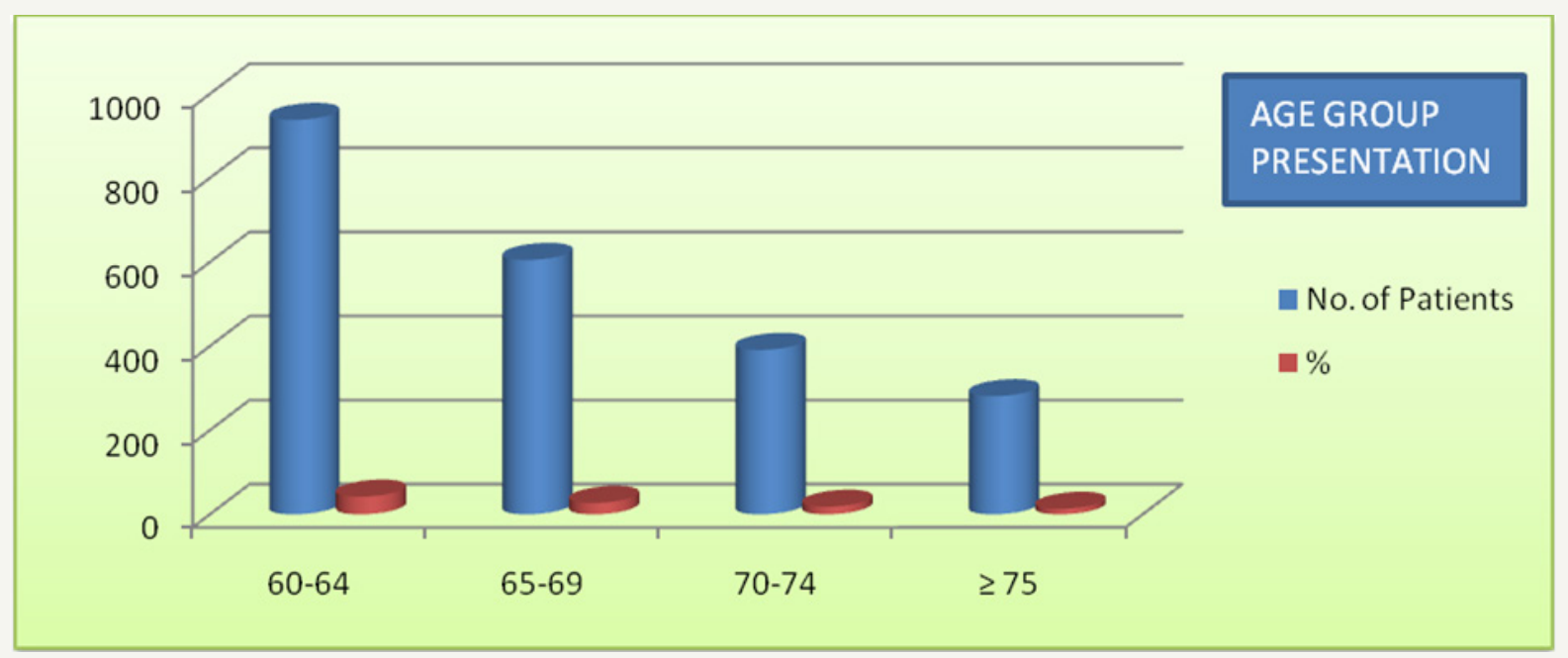

Figure 1: Age Group Presentation.

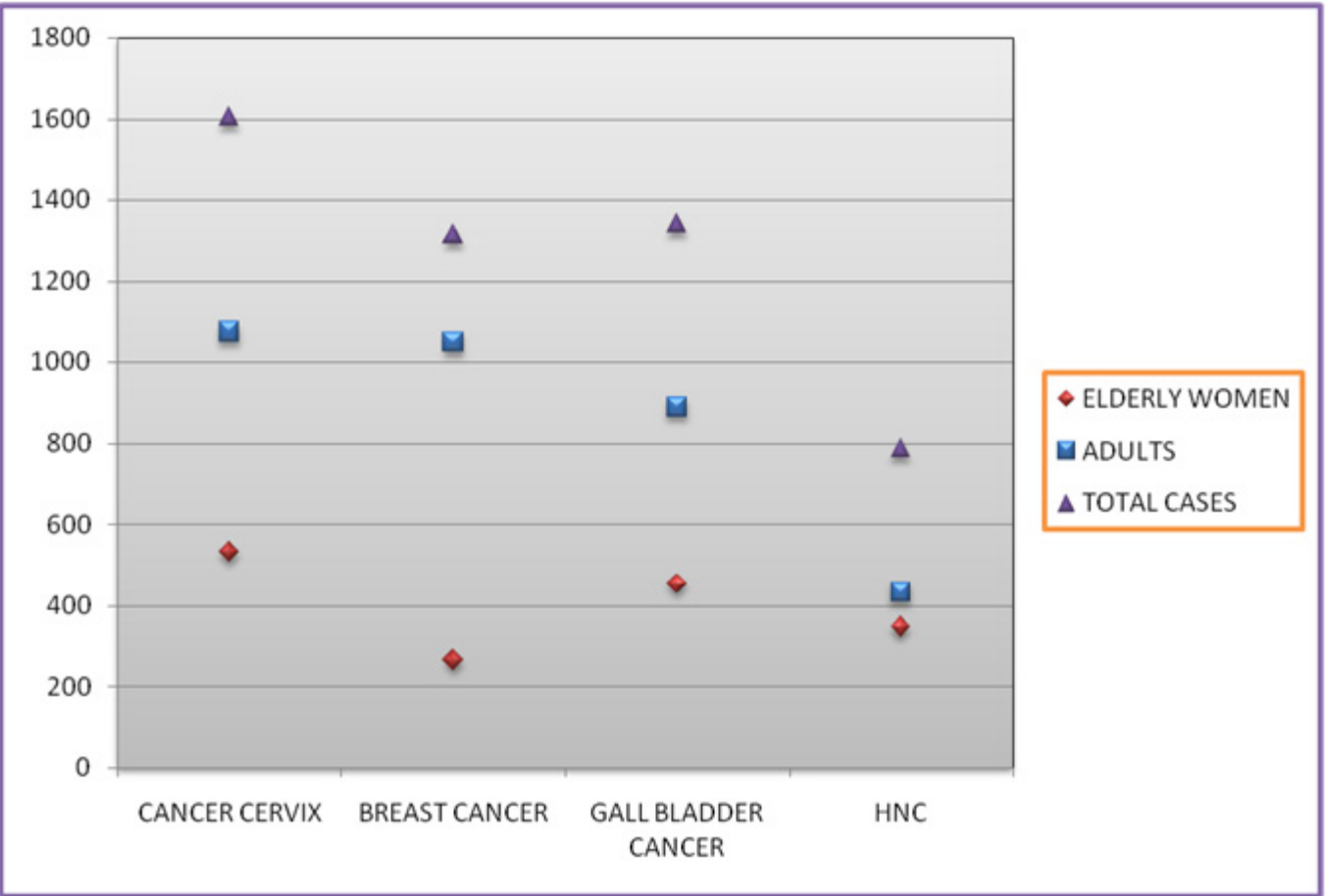

Figure 2: Comparisons Of Disease Pattern between Elderly Women ( $\geq 60$ years) \& Adults (20-59 years).

Table abbreviations: HNC-Head and Neck Cancers.

The total number of cancer cases in elderly women accounted for $31.25 \%$ as per the hospital based registry during the 3 year study period. The most common age group affected with the disease was 60-64 years (Figure 1). The most common cancer sites were 
cervix (23.9\%), followed by gall bladder (20.45\%), head and neck (15.66\%), breast (12.18\%), ovary (4\%) and lung (3.5\%). In contrast, adults of age group between 20-59 years has shown different pattern of the disease with cancer cervix $(22.7 \%)$ being the most common malignancy followed by breast cancer (22\%), gall bladder cancer (18.8\%), HNC (9.3\%) (Figure 2). Among HNC, buccal mucosa cancers were commonly encountered $(41.7 \%)$ and nasopharyngeal cancers were least encountered $(0.8 \%)$. Interestingly, gall bladder cancer is the $2^{\text {nd }}$ most common malignancy overall in the females (18.93\%), and has surpassed breast cancer (18.55\%) in the region.
The socio-demographic profile was recorded and found most of them hailed from rural India (74\%), uneducated (89\%) and lowmiddle income group (87\%). Most of the patients presented with advanced stages especially in gall bladder cancer, where metastatic disease outnumbered any disease among elderly population (Figure 3). Most of the participants has limited knowledge and misconceptions due to poor awareness. $75 \%$ of the participants believed that life after cancer is worthless; $81 \%$ viewed that cancer therapy is painful and unsuccessful; and $79 \%$ people felt cancer cannot be diagnosed early.

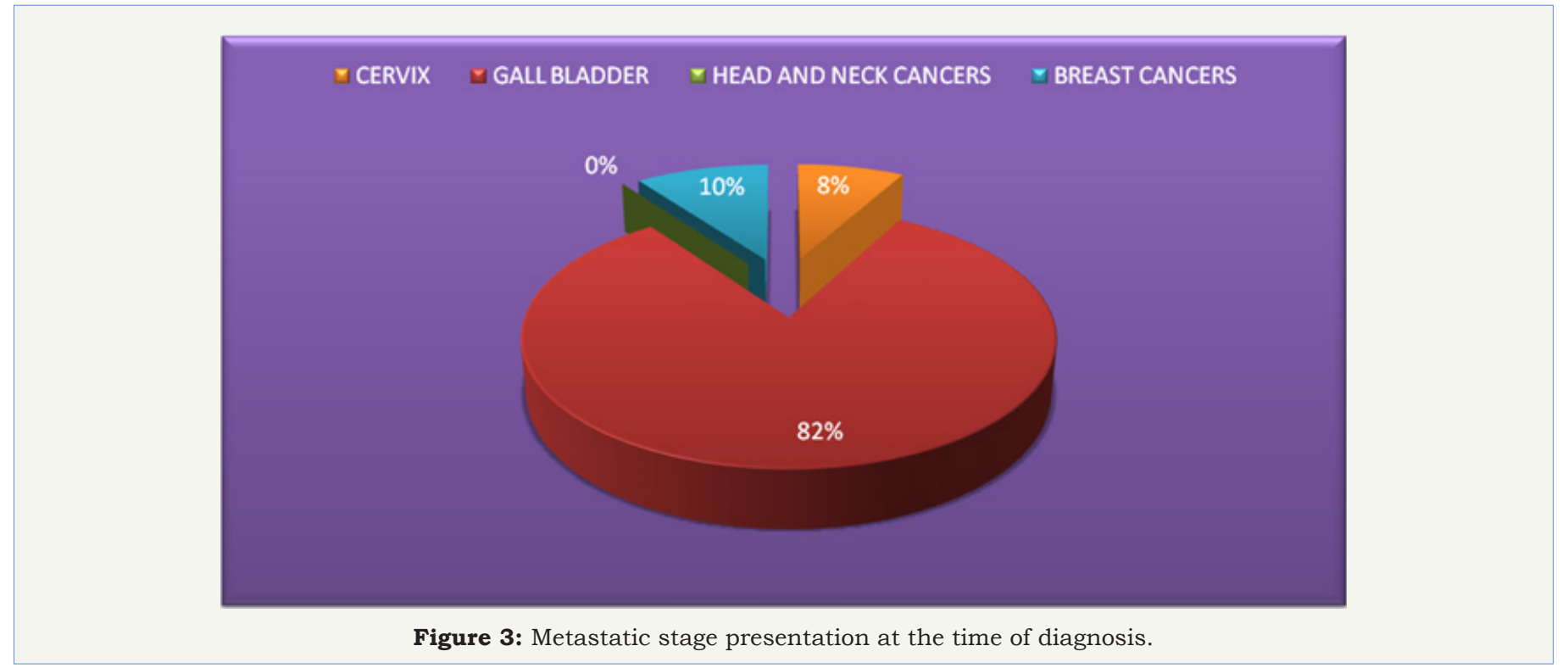

\section{Discussion}

The scientific paper has discussed the cancer trends in elderly women reported to Regional Cancer Centre, Allahabad, India. As per analysis of census data and projections, elderly population sex ratio is in favor of female elderly [5]. The health infrastructure should blend with the cancer trends for successful detection and awareness camps. As per the hospital cancer registry, cancer cervix was the most commonly encountered among elderly population which agrees with other reports [6]. In Indian scenario, most of the women do visit the physicians unless they face any symptoms such as bleeding/pus or water discharge/vaginal pain/bleeding during intercourse as in case of cervical carcinoma. Pap smear screening has been advocated but still the outlooks of the people need to be addressed seriously. Gall bladder cancer is the second most common cancer leading to mortality and morbidity in the region which is scarce in other regions of the world [7]. The incidence and mortality rates do vary geographically in Indian subcontinet where most of the cases were documented along the Indo-Gangetic belt region in Northern part of India. The risk factors could be the pollutants in the water/lifestyle/socio-demographic parameters, where statistical correlation has been not established yet. Further research is required in this domain to face future challenges. The trend of breast cancer has shifted towards adults of age $\leq 60$ years citing to early detection and awareness at community level [8]. Smokeless tobacco consumption is rampant in the region either in the form of pan, gutkha, supari, manjan, betel leaf etc., made HNC as the 3rd most common cancer in the region in the elderly women. The oral cancer burden varies geographically within India and more well differentiated studies and research should focus in this domain [9]. Framing a more balanced health infrastructure, reaching out to people at community level, primary health centers, and local clinics will certainly change the scenario to a better outcome in rear future [10].

\section{Conclusion}

The research emphasized the most sensitive group of population viz., elderly women with cancer. The elderly women outlook towards life changes through different phases of journey and this impact various domains including health. The elderly population requires back up and support in every phase of cancer journey as it is very tedious process. To fight against the cancer, one should be armed with courage, strength, dedication, patience and optimism. This is possible only through the support from family and friends. The pattern of the disease among elderly women is different from the adults of age group between 20-59 years, and the screening and detection camps should configure the strategies accordingly. Educating the people at community level is vital, and many camps/ events/programmes have been organized nationally and locally in India but still the outlook of the people ought to change. Door- door campaigns, pamphlets, TV shows/stage performances in malls/ 
public gatherings/voluntary participation will definitely change the current scenario and it is a long way uphill task which needs cooperation and perseverance from the people. The limitation of the study was a single institution based experience and collaboration with multiple cancer registries, hospitals and tertiary centers' nationwide will help in designing detection camps and awareness programmes meticulously.

\section{Future Implications}

Based on the cancer trends in elderly women, the screening and cancer awareness programmes can be framed meticulously and for any future Oncology department set up in the state can focus on the trends and devise plans accordingly for the welfare of the society. Gall bladder cancer is the 2nd most common malignancy in the region, which is scarce in other regions of the world. Further research in this domain for early detection will reduce the mortality rates significantly. Breast cancer is no more a disease of elderly population owing to the successful detection camps and educating at community level. Early stages improve the overall survival and enhance quality of life. Head and neck cancers (HNC) which are mostly tobacco related especially smokeless tobacco which is rampant in the region, can be reduced by educating the society and implementing strict laws against tobacco chewing and selling. The article can be helpful for potential future research and directions.

\section{References}

1. (2011) Government of India. National Programme for the Health Care of the Elderly (NPHCE), Operational guidelines. Government of India, New Delhi, India.

2. (2008) United Nations Department of Economic and Social Affairs, Population Division. World Population Prospects.

3. WHO (2016) Definition of an older or elderly person.

4. (2012-2014) Population Based Cancer Registries (PBCR). ICMR Report.

5. (2006) Report of the technical group on population projections constituted by the National Commission on Population, published by the office of the registrar general, India.

6. Nandakumar A, Ramnath T, Chaturvedi M (2009) The magnitude of cancer cervix in India. Indian J Med Res 130(3): 219-221.

7. Randi G, Franceschi S, La Vecchia C (2006) Gallbladder cancer worldwide: geographical distribution and risk factors. Int J Cancer 118(7): 15911602.

8. Murthy NS, Agarwal UK, Chaudhry K, Saxena S (2007) A study on time trends in incidence of breast cancer-Indian scenario. Eur J Cancer Care 16(2): 185-186.

9. Manoharan N, Tyagi BB, Raina V (2010) Cancer incidences in rural Delhi-2004-05. Asian Pac J Cancer Prev 11(1): 73-77.

10. Goodwin JS, Samet JM, Hunt WC (1996) Determinants of survival in older cancer patients. J Natl Cancer Inst 88: 1031-1038. 